\title{
A piecewise geometric analysis method for real-time ambulatory ECG detection
}

\author{
H.-Y. Zhou ${ }^{\mathrm{a}, \mathrm{b}}$, J. Li ${ }^{\mathrm{a}} \mathrm{b}, *$, D.-C. Zuo ${ }^{\mathrm{b}}$, K.-M. Hou ${ }^{\mathrm{c}}$ and C. de Vaulx ${ }^{\mathrm{c}}$ \\ ${ }^{a}$ School of Electrical and Information Engineering, HuBei University of Automotive Technology, \\ Shiyan, HuBei, China \\ ${ }^{\mathrm{b}}$ School of Computer Science and Technology, Harbin Institute of Technology, Harbin, Heilongjiang, \\ China \\ ${ }^{\mathrm{c}}$ LIMOS Laboratory CNRS UMR, University of Blaise Pascal, Clermont-Ferrand, France
}

\begin{abstract}
.
BACKGROUND: As one of the pervasive healthcare services, Ubiquitous cardiac care (UCC) systems should have at least two significant characteristics: real-time detection capability for cardiac arrhythmia events and a small resource requirement for its computation and storage.

PURPOSE: Due to the strict-constrained system support and ambulatory signal quality in the out-of-hospital pervasive healthcare applications, a dedicated real-time AED (Ambulatory Electrocardiograph Detection) algorithm has been implemented. METHODOLOGY: By adopting the piecewise geometric analysis method, this algorithm can provide a real-time continuous detection capability for QRS complexes, which consists of three main functional modules: the Data preparation; the R-wave vertex discovery; and the QRS complex recognition. Currently, this algorithm has been applied on an on-line UCC application system at the hospital for more than 30 patients.

RESULT: The performance evaluation has been made not only on the standard MIT-BIH cardiac arrhythmia database but also on the clinical testing. The experiential results explore this algorithm has in average sensitivity of $99.37 \%$ and specificity of $99.72 \%$.

CONCLUSION: This AED algorithm has minimal beat detection latency and a less computation consumption, which make it meet the requirements of ubiquitous cardiac care applications.
\end{abstract}

Keywords: Ubiquitous cardiac care, ambulatory ECG, piecewise geometric analysis, QRS complex detection

\section{Introduction}

Owning to the technology development in mobile computing and Internet of Things, pervasive healthcare is becoming a novel health care fashion, which can provide an out-of-hospital, long-term, real-time intelligent healthcare services. However, the emergence of ubiquitous care services also brings about some new technical challenges, such as the reliability and complexity issues of the detection algorithms for the physiological abnormal events, especially when taking into account of the resource-limited care devices and the complex care situations [1].

\footnotetext{
${ }^{*}$ Corresponding author: Jian Li, School of Electrical and Information Engineering, HuBei University of Automotive Technology, N.167, CheCheng West Road, Shiyan, HuBei 442002, China. Tel.: +86 719823 8200; Fax: +86 719 8239071; E-mail: lijian@ftcl.hit.edu.cn.
}

0928-7329/15/\$35.00 (C) 2015 - IOS Press and the authors. All rights reserved

This article is published online with Open Access and distributed under the terms of the Creative Commons Attribution NonCommercial License. 
Ubiquitous cardiac care (UCC) is one of pervasive healthcare services, which needs a dedicated realtime AED (Ambulatory Electrocardiograph Detection) algorithm that should have at least two significant characteristics: real-time detection capability for cardiac arrhythmia events; a small resource requirement for its computation and storage. As the body-surface manifestation $(m V)$ of cardiac electrical potentials, QRS ( $Q-R-S$ waves) complexes in ECG signals have a remarkable waveform shape (high potential amplitude, steep slope) and plentiful waveform information, which are thus utilized in AED algorithms as an essential basis for QRS complex detection.

According to the analysis methodologies, classical AED algorithms are mainly classified as [2]: Temporal domain; Wavelet transform; Syntax analysis; Neural network; etc. In recent years, some hybrid AED technologies have been introduced, which improve the detection accuracy but result in more computation complexity and resource consumption [3]. Due to the strict-constrained system support and ambulatory ECG signal quality, current studies on AED technologies mainly address clinic testing but rarely ubiquitous care applications. In this paper, a real-time AED algorithm dedicated to UCC system is proposed, shown in Section 2. In Section 3, the algorithm performance is evaluated. Finally, the conclusions are drawn in Section 4.

\section{AED algorithm}

Owing to the less computational overload in the temporal domain analyses, a piecewise geometric realtime QRS detection algorithm is proposed, which includes three main modules: the Data preparation that aims to provide the de-noised and normalization signals; the $R$-wave vertex discovery that aims to detect $R$-wave vertices in consecutive data segments; and the QRS complex recognition that aims to identify QRS complexes based on the $R$-wave vertex-pair.

\subsection{Data preparation}

In general, the original $A E C G$ signals $R(t)$ are non-stationary and noise-sensitive, the main interferences of which are: the electrical noises, the baseline drifts, the muscle tremor noises and the motion artifacts. In order to reduce noises and remove interferences, several classical signal preprocessing techniques are adopted together, which includes the normalization operation, the differentiator and multiple classical filters. This module generates two signal series, i.e. the self-adaptive differential signals $A D(t)$ for the QSR complex detection, and the high quality filtering signals $R C(t)$ for the complex feature extraction and arrhythmias classification, shown in Fig. 1, more details see in [4].

\section{2. $R$-Wave vertex discovery}

The $R$-wave of QRS complexes on $A D(t)$ have a rapid change of waveforms and a high amplitude of electrical potentials, which are used to detect QRS complexes. Besides, in view of the computation complexity, the $A D(t)$ signals are divided into a set of data segments with a 5-second length. In general, the $R$-wave of QRS complexes has one vertex-pair, which consists of a positive vertex and a negative one, which can be identified as the first-order derivative zero-crossing points, i.e. the largest absolute potential points on $A D(t)$.

Three reference lines, i.e. the baseline in which $A D(t)=0$, the positive threshold line $P T$ and the negative threshold line $N T$, are used to estimate the $R$-wave vertexes. In Table 1, the self-adaptive threshold 
Table 1

Algorithm of $R$-wave vertex discovery

//Divide a 5 s segment into five 1 s sub-segments, then $N=\{0 \ldots 4\}$;

//Detect the Max and Min sample points in each sub-segment and then Obtain the set of vertex-pair

1. Let $\operatorname{Vpos}(i)=\operatorname{Max}(A D(t)), \operatorname{Vneg}(i)=\operatorname{Min}(A D(t)) ; t \in[i * 500:(i+1) * 500-1], i \in N, n=N, K=\emptyset$;

//Calculate the means of positive and negative vertexes in the $5 \mathrm{~s}$ segment.

2. Let $\operatorname{Mpos}=\sum \operatorname{Vpos}(i) /(n+1) ; \operatorname{Mneg}=\sum \operatorname{Vneg}(i) /(n+1) ; i \in N \& \& i \notin K$;

//Exclude the artificial vertexes into $K$ set and Redo step $(2,3)$ until no artifact exists.

3. While $(i \in N \& \& i \notin K)$

If $(\operatorname{Vpos}(i) \gg \operatorname{Mpos} \| \operatorname{Vneg}(i) \ll \operatorname{Mneg})$ Then $n--, i \in K$; Goto (2);

$/ /$ Calculate the threshold values $(P T, N T)$ of the vertex-pair in the 5 s segment, set $\rho=0.45$ after studying.

4. Let $P T_{c u r}=\rho * \operatorname{Mpos} ; N T_{c u r}=\rho * \operatorname{Mneg}$;

//Estimate threshold values (PT, NT) by comparing it with the threshold of the previous segment

5. If ( $\left|P T_{\text {cur }}-P T_{\text {pre }}\right|$ or $\left|N T_{\text {cur }}-N T_{\text {pre }}\right|>$ estimated threshold $)$ Then $P T_{\text {cur }}=P T_{\text {pre }}, N T_{\text {cur }}=N T_{\text {pre }}$; Else $P T_{\text {cur }}=\left(P T_{\text {pre }}+P T_{\text {cur }}\right) / 2, N T_{\text {cur }}=\left(N T_{\text {pre }}+N T_{\text {cur }}\right) / 2$.

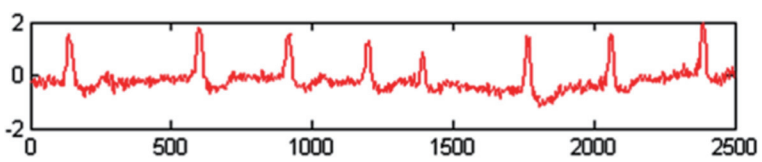

(a) $R(t)$

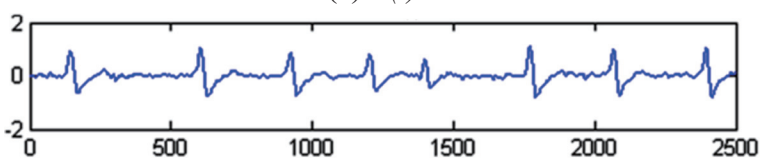

(b) $A D(t)$

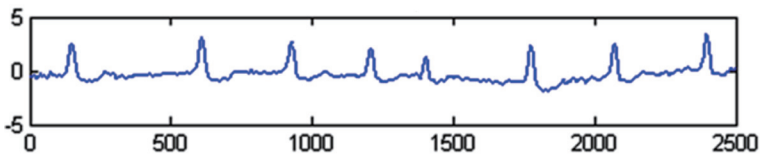

(c) $R C(t)$

No. of samples / dot

Fig. 1. Data preparation: (a). the raw AECG signal $R(t)$; (b). the self-adaptive differential filtering signal $A D(t)$; (c). the standard de-noised filtering signal $R C(t)$.

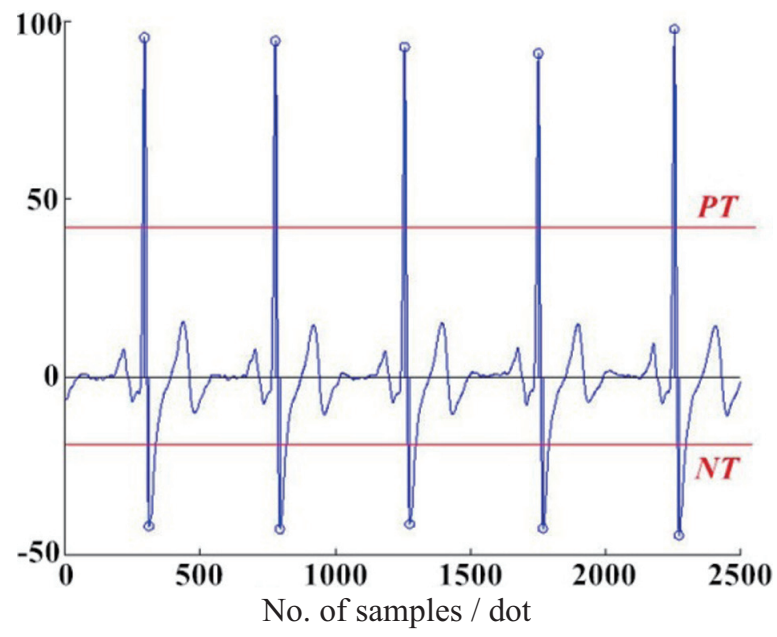

Fig. 2. Estimation of the vertex-pairs within a $5 \mathrm{~s}$ segment based on the self-adaptive threshold estimation.

estimation mechanism for the $R$-wave vertex discovery in the $5 \mathrm{~s}$ data segment is presented. In this module, the $5 \mathrm{~s}$ data segment is divided into five $1 \mathrm{~s} \mathrm{sub}$-segments, which is based on the basic fact that the normal heart rate of a healthy adult is $60-100 \mathrm{bmp}$. A set of vertex-pair can be thus obtained for each segment, which is used to locate the QRS complexes on $A D(t)$, illustrated in Fig. 2.

\subsection{QRS complex recognition}

The data structure termed $Q R S \_C O M P L E X$ is defined to record the information of a QRS complex, shown in Fig. 3, which includes the class information of QRS complex, i.e. etype, and the position information of some key points, i.e. the positions of onset and end points (tstart, tstop), the vertexes' positions (tpic1, tpic2) and their voltage values (Vpic1, Vpic2), and the raw signal date of the complex stored in complex. 


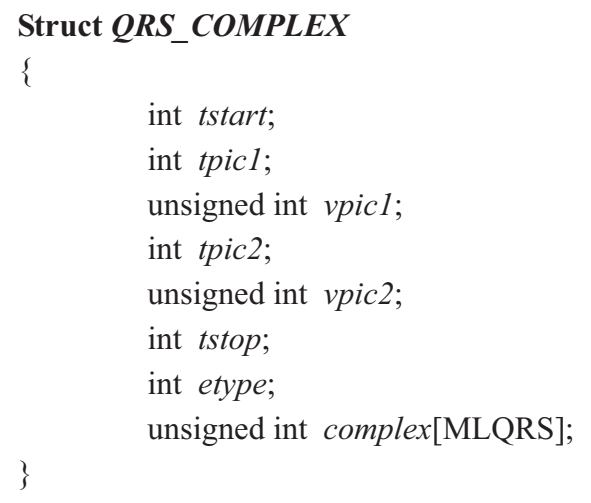

Fig. 3. Data structure of QRS complex: QRS_COMPLEX.
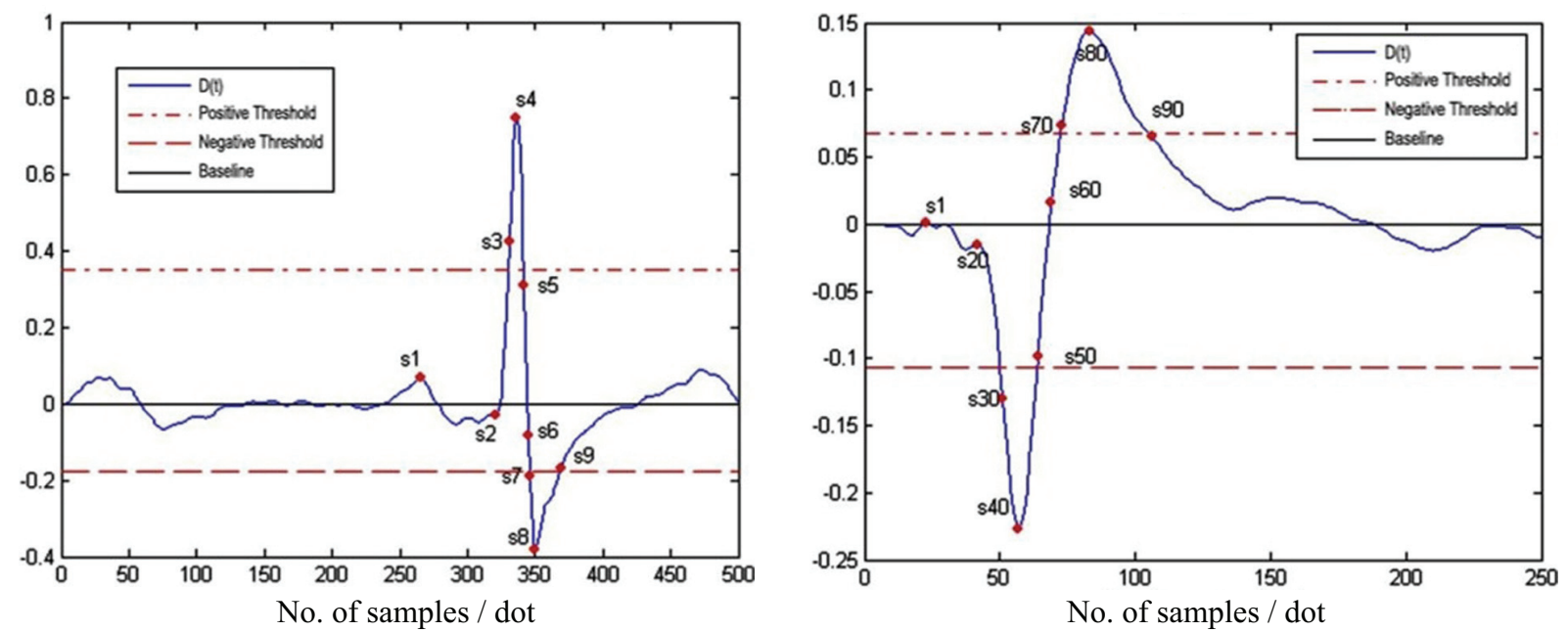

Fig. 4. Different state set of QRS complexes: positive complex (left); negative complex (right).

In general, the QRS complexes can be classified into two categories: positive and negative. Correspondingly, two groups of states are defined to illustrate the morphological characteristics of different QRS complexes based on the three reference lines, which are the positive state set defined as $\{S 2, \ldots$, $S 9\}$, and the negative state set defined as $\{S 20, \ldots, S 90\}$, depicted in Fig. 4.

Table 2 illuminates the state definitions and state transition rules for the normal positive QRS complexes. It should be noted that the negative one is just the reverse. The finite-state-machine model for the QRS complex recognition is shown in Fig. 5, where $S 1$ is the initial \& end state.

Some exceptional conditions may occur due to the improper or inaccurate QRS recognitions resulting from the pseudo-complexes or the abnormal complexes by reasons of the interferences or the cardiac diseases. In view of these abnormal cases, this algorithm proposes an exceptional handling mechanism: one counter termed cptr is defined, which is initial to zero when entering a new state, i.e. $c p t r==0$, and will increase one automatically when each time processing a sample point, i.e. $c p t r++$. If $N o$ state transition condition is satisfied when the cumulative sample number is beyond the maximum estimated threshold value $(M D x)$, i.e. cptr $>M D x$, therefore we can suppose that the next state doesn't exist and then the following operations will be performed according to the current state of this complex. 


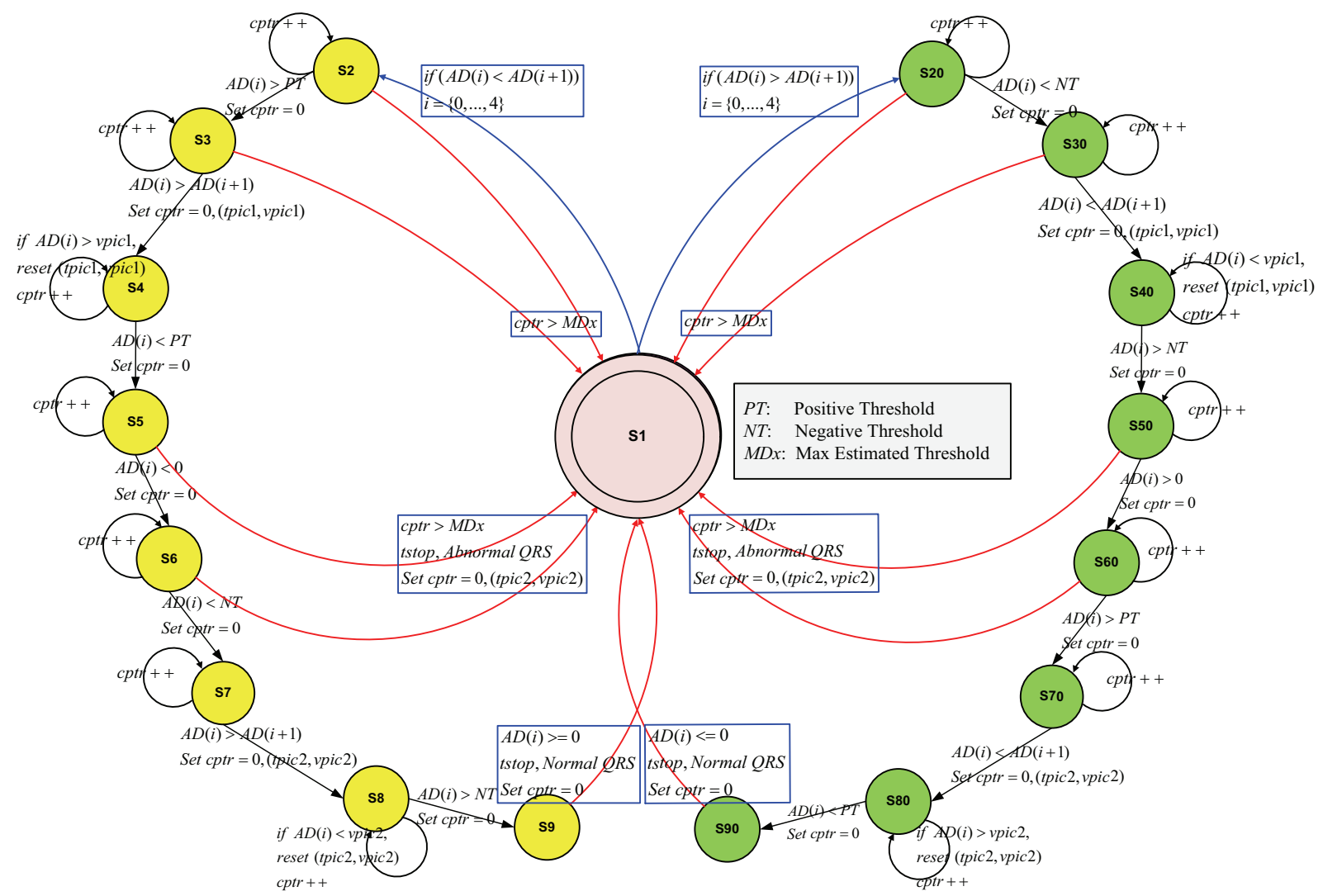

Fig. 5. Finite-state-machine model for $Q R S$ complex recognition.
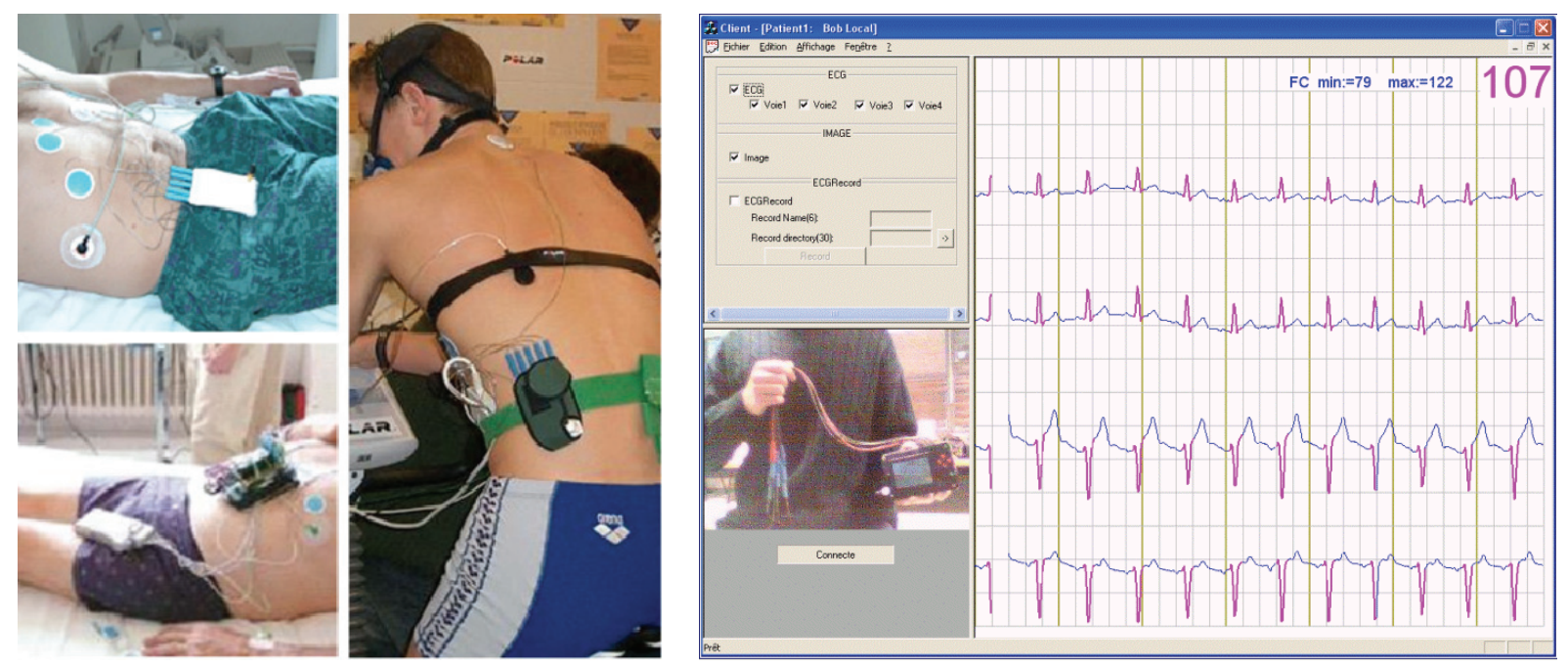

Fig. 6. Application illumination of this algorithm on a UCC system. 
Table 2

Algorithm of QRS complex recognition

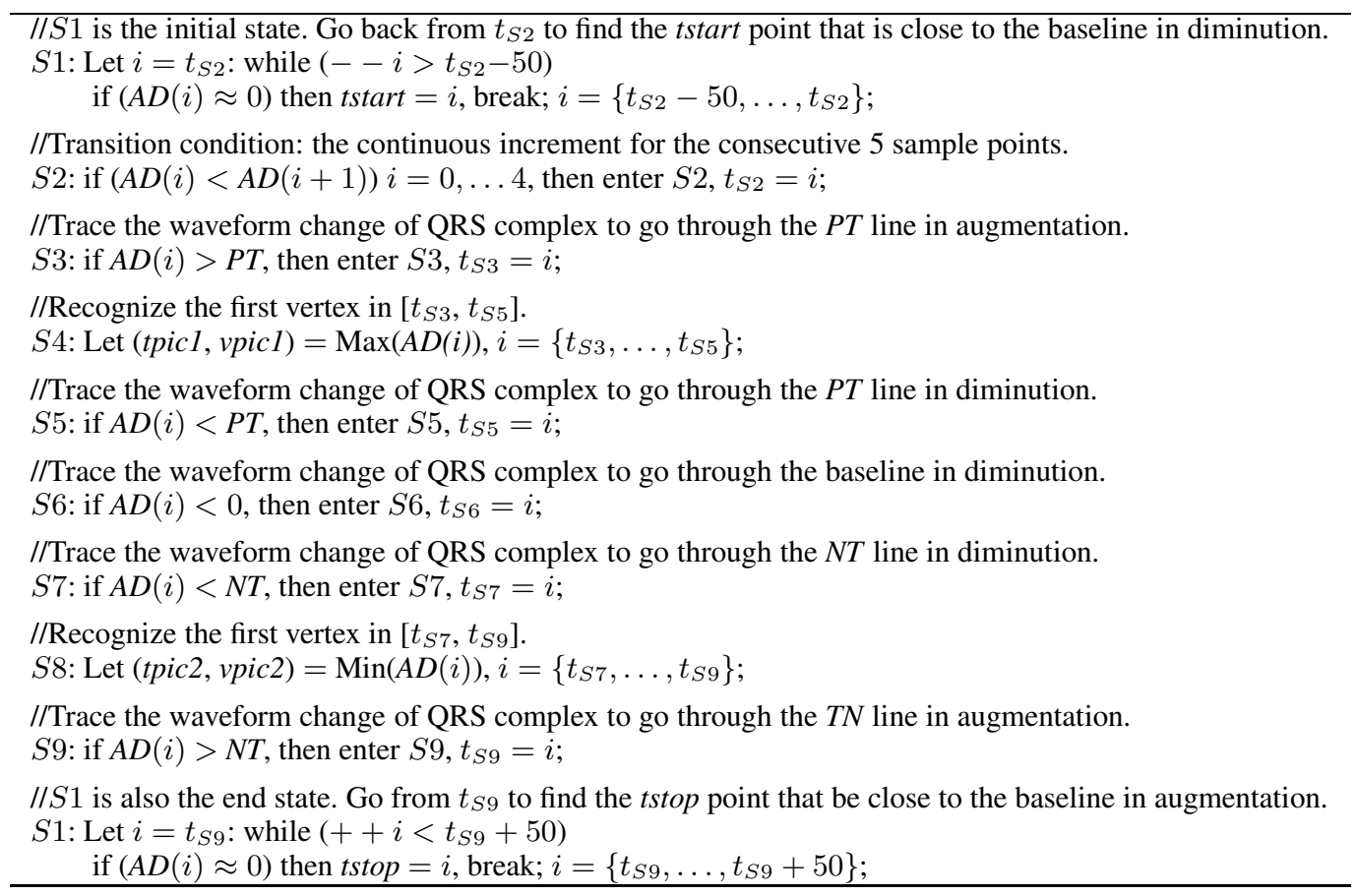

- If (tpicl, vpicl) is not selected on the previous phases, then we can suppose this complex is the pseudo-one and the complex state will be set back to $S 1$ direcly.

- If (tpicl, vpicl) is selected, then we suppose this complex is the abnormal one and the tstop will be set before entering $S 1$.

\section{Experimental results and performance evaluations}

Currently, this algorithm has been evaluated on two different ECG databases: MIT-BIH arrhythmia database [5] and CSD database (Clinic STAR Database). The former contains 48 half-hour excerpts of two-channel ambulatory ECG recordings, and the latter is obtained from the clinical testing on more than 30 patients at the Gabriel Montpied hospital (CHRU de Clermont-Ferrand, France) by adopting a UCC system in real-time [6], shown in Fig. 6.

Dotsinsky et al. [10] defined four performance indexes to evaluate the algorithm efficiency (Se: sensitivity and $S p$ : specificity): $T P$ (true positive), $F P$ (false positive), $F N$ (false negative) and shifted $S H$ beats, shown in Eq. (1). The $S H$ beat, representing a signal fault, is assigned to a couple of adjacent erroneously detected $F P$ and $F N$ in case they are preceded and followed by the $T P$ beats.

$$
S e \frac{T P}{T P+F N+S H} \quad S p=1-\frac{F P}{T P+F P}=\frac{T P}{T P+F P}
$$

Table 3 shows the performance evaluation on the sensitivity $(S e)$ and specificity $(S p)$ of this algorithm. The performance results of this detection algorithm, $99.06 \%$ sensitivity and $99.69 \%$ specificity on the MIT-BIH daTables $99.67 \%$ sensitivity and $99.74 \%$ specificity on the CSD database, show its high sensitivity and specificity. Besides, this detection algorithm has minimal beat detection latency, low computational consumption and fast detection ability. 
Table 3

Performance evaluations and comparisons

\begin{tabular}{lll}
\hline AED algorithms & Se $(\%)$ & Sp $(\%)$ \\
\hline Tabakov et al. [7] & 99.37 & 99.51 \\
Afonso et al. [8] & 99.59 & 99.56 \\
Poli et al. [9] & 99.60 & 99.51 \\
Dotsinsky and Stoyanov [10] & 99.04 & 99.62 \\
Kaiser and Findeis [11] & 99.68 & 99.72 \\
Datex-Ohmeda Corp. [12] & 99.86 & 99.88 \\
Millet et al. [13]: alg.1/alg.2 & $94.6 / 97.3$ & $98.0 / 98.0$ \\
Chiarugi et al. [14] & 99.76 & 99.81 \\
Elgendi et al. [15]: alg.1/alg.2 & $87.9 / 97.5$ & $97.6 / 99.9$ \\
This AED alg.: MIT/CSD & $99.06 / 99.67$ & $99.69 / 99.74$ \\
\hline
\end{tabular}

\section{Conclusion}

This work aims to design a household/clinical cardiac arrhythmias monitoring system that can provide the capability of real-time QRS complex detection. In view of the strict-constrained system resource and ambulatory signal quality in UCC applications, a dedicated real-time continuous AED algorithm is proposed, which adopts the piecewise geometric method to detect QRS complexes from Ambulatory ECG signals. Currently, this algorithm has been applied on a UCC system. The performance evaluations have been done on the MIT-BIH cardiac arrhythmias database and the patients' records of the hospital of CHU de Clerment-Ferrand (France). The experiential results explore this algorithm has in average sensitivity of $99.37 \%$ and specificity of $99.72 \%$. Furthermore, this algorithm has minimal beat detection latency and a less computation consumption, which make it meet the requirements of UCC applications.

\section{Acknowledgements}

The authors would like to thank all the colleagues and copartners who have contributed to the study, and also are grateful for the Natural Science Foundation of HUAT (BK201411), the Sci-technical Pillar program of Hubei Province (2014BHE024), and the Provincial Science Foundation of China (2014CFB632) for their supports to this project.

\section{References}

[1] Sneha S, Varshney U. Enabling ubiquitous patient monitoring: Model, decision protocols, opportunities and challenges, Decision Support Systems, 2009, vol.46, pp.606-619.

[2] Köhler BU, Hennig C, Orglmeister R. The principles of software QRS detection, IEEE Engineering in Medicine and Biology Magazine, 2002, vol.21(1), pp.42-57.

[3] Huang WX, Chen JH, Huang BQ, Wang FY. Study on feature extraction for ECG beat classification, Biomedical Engineering and Clinical Medicine, 2007, vol.11(5), pp.344-347 (in Chinese).

[4] Zhou HY. Réseau de capteurs sans fil dédié à la détection et au diagnostic d'arythmie cardiaque en temps réel, en continu et à distance, Ph.D. thesis, University of Blaise Pascal, Clermont-FD, France, Nov. 2004.

[5] Moody GB, Mark RG. The MIT-BIH arrhythmia database on CD-ROM and software for use with it, Computers in Cardiology, 1990, vol.(17), pp.185-188.

[6] Li J, Zhou HY, Zuo DC, Hou KM, De vaulx C. Ubiquitous health monitoring and real-time cardiac arrhythmias detection: A case study, Bio-Medical Materials and Engineering (BMME), 2014, vol.24(1), pp. 1027-1033.

[7] Tabakov S, Iliev I, Krasteva V. Online digital filter and QRS detector applicable in low resource ECG monitoring systems, Annals of Biomedical Engineering, 2008, vol.36(11), pp.805-1815. 
[8] Afonso VX, Tompkins WJ, Nguyen TQ, Luo S. ECG beat detection using filter banks, IEEE Transaction on Biomedical Engineering, 1999, vol.46, pp.192-202.

[9] Poli R, Cagnoni S, Valli G. Genetic design of optimum linear and nonlinear QRS detectors, IEEE Transaction on Biomedical Engineering, 1995, vol.42, pp.137-141.

[10] Dotsinsky IA, Stoyanov TV. Ventricular beat detection in single channel electrocardiograms, BioMedical Engineering OnLine, 2004, vol.3(3).

[11] Kaiser W, Findeis M. Novel signal processing methods for exercise ECG, special issue on electrocardiography in ischemic heart disease, International Journal of Bioelectromagnetism, 2000, vol.2(1), pp.61-65.

[12] Datex-Ohmeda Corp. Bedside arrhythmia monitoring quick guide, Education Report, Internal web journal for medical professionals Cardiovascular. 2002(11), http://www.clinicalwindow.com.

[13] Millet J, Perez M, Joseph G, Mocholi A, Chorro J. Previous identification of QRS onset and offset is not essential for classifying QRS complex in a single lead, Computers in Cardiology, 1997, vol.24, pp.299-302.

[14] Chiarugi F. Adaptive. Threshold QRS detector with best channel selection based on a noise rating system, Computers in Cardiology, 2007, vol.4, pp.157-160.

[15] Elgendi M. A robust QRS complex detection algorithm using dynamic thresholds, International Symposium on Computer Science and its Applications, 2008, pp.153-158. 